\title{
Zoneamento agroclimático para a cultura do pinhão-manso no Estado de Pernambuco ${ }^{1}$
}

\author{
José M. C. Possas ${ }^{2}$, Marcus M. Correa ${ }^{2}$, Geber B. de A. Moura ${ }^{3}$, \\ Pabrício M. O. Lopes ${ }^{3}$, Anildo M. Caldas ${ }^{2}$ \& Robertson V. de P. Fontes Júnior ${ }^{2}$
}

\section{RESUMO}

Propõe-se, com o presente trabalho, realizar o zoneamento agroclimático da cultura do pinhão-manso (Jathopra curcas L.) no estado de Pernambuco, motivo pelo qual foram utilizados dados climáticos de temperatura do ar média e precipitação pluviométrica de 146 postos meteorológicos, dos quais 73 com série histórica de dados acima de 30 anos e outros 73 com série abaixo de 30 anos porém maior que 20 anos de observações. A escolha do pinhão-manso foi motivada pelo potencial desta cultura como fonte de energia renovável em substituição aos combustíveis fósseis, tanto quanto pela sua rusticidade, tornandose uma nova opção para regiões de clima semiárido, como ocorre em grande parte da região Nordeste. A seleção da região de estudo está relacionada à variabilidade climática apresentada por esse estado e ao potencial que a referida cultura tem em se desenvolver em regiões nas quais a chuva é escassa. De acordo com o estudo, o Estado de Pernambuco apresenta $14,92 \%$ de áreas aptas ao cultivo do pinhãomanso, 44,26\% de áreas restritas por deficiência hídrica e 40,82\% de áreas inaptas.

Palavras-chave: aptidão climática, biocombustíveis, krigagem

\section{Agroclimatic zoning for physic mut crop in the State of Pernambuco}

\begin{abstract}
This study aimed to realize the agroclimatic zoning of Jathopra curcas L. crop in the State of Pernambuco. For this, climatic data of air temperature and mean rainfall of 146 meteorological stations and 73 series with historical data over 30 years and another 73 with less than 30 years but more than 20 years of observations were used. The choice of Jatropha has been motivated by the potential of this crop as a source of renewable energy to replace fossil fuels as well as for its rusticity and a new option for regions of semi-arid climate, as it occurs in the Northeast region. The selection of the study area is related to climate variability in the State and the potential that this crop has in regions where there is low precipitation. According to the study, the State of Pernambuco has $14.92 \%$ of areas suitable for production of Jatropha, $44.26 \%$ of the restricted areas by water deficit, and $40.82 \%$ of unsuitable areas.
\end{abstract}

Key words: suitability climate, biofuels, kriging, Jathopra curcas L. 


\section{INTRODUÇÃO}

O mundo tem grande interesse na produção de biocombustíveis em função da crescente exigência pela redução da emissão de gases poluentes, que resultam em acordos como o Protocolo de Kyoto. Outra variável que exerce pressão por uma nova fonte de energia alternativa ao combustível fóssil, é a instabilidade política e social nos países produtores de petróleo, principalmente no Oriente Médio (Lima Filho et al., 2008).

Com a preocupação atual com o efeito estufa, o aquecimento global e a escassez das reservas mundiais de combustível fóssil, o pinhão-manso tem despertado interesse dos produtores, do governo e das instituições de pesquisa. Desta forma e com a possibilidade do uso do óleo do pinhão-manso para a produção do biodiesel, abrem-se amplas perspectivas para o crescimento das áreas de plantio com esta cultura, no semiárido nordestino (Arruda et al., 2004).

A produção de biodiesel no Brasil vem se expandindo desde que a utilização do referido biocombustível em mistura com o diesel mineral se tornou compulsória, passando de 404 milhões de litros em 2007 (Ambrozin et al., 2009) para 2,64 bilhões de litros em 2011 (ANP, 2012).

Os resultados do Programa Nacional de Produção de Biodiesel (PNPB), lançado em 2005, apontam o predomínio da soja como principal matéria-prima o que, para Roscoe et al. (2007), atrela o biodiesel à competição com o mercado de alimentos o que, sem dúvida, acarretaria um padrão de produção em grandes extensões de terra, dificultando a participação da produção familiar.

Uma potencial cultura para produção de biocombustível que não participa de cadeias de produção voltadas para alimentação humana e animal, é o pinhão-manso (Jatropha curcas L.), oleaginosa que vem sendo considerada uma das espécies mais promissoras para a produção de biodiesel no Brasil visto que as características do óleo produzido por suas sementes apresentam boa semelhança com o diesel do petróleo. Sato et al. (2007) destacam que o pinhão-manso se tornou matériaprima atrativa para produção de biodiesel por apresentar elevado potencial de rendimento de grãos e óleo.

Andrade et al. (2007) salientam que, anualmente, a cultura do pinhão-manso produz no mínimo 2 toneladas de óleo por hectare, variando de acordo com a região de plantio, método de cultivo, tratos culturais, idade da cultura, quantidade de chuva e fertilidade do solo. Drumond et al. (2010) encontraram, ao avaliar 10 genótipos de pinhão-manso sob condições de irrigação e adubação no município de Santa Maria da Boa Vista, Sertão do São Francisco, Pernambucano, produtividades de sementes entre 2853 e $3542 \mathrm{~kg} \mathrm{ha}^{-1}$, em plantas com 12 meses de idade; apesar disto, em condições de sequeiro as pesquisas no Brasil, quanto à produtividade do pinhão-manso, ainda são incipientes e preliminares.

Segundo Sato et al. (2007) a cultura do pinhão-manso pode ser considerada opção agrícola para áreas áridas e semiáridas, na recuperação de áreas degradadas, promove a integração do acesso à produção com renda (pela da venda do óleo das sementes para fins combustíveis), é suprimento de energia (o óleo pode ser utilizado em motores e máquinas para a geração de eletricidade), contribui para o desenvolvimento rural (com o emprego da mão-de-obra familiar) e com consequente fixação do homem no campo e segurança alimentar, visto que permite o uso de culturas anuais alimentícias em consórcio, além de melhorias ambientais, como a formação de um microclima que favorece o desenvolvimento de outras culturas nas entrelinhas, entre outros. Entretanto, a espécie ainda se encontra em processo de "domesticação" e, conforme Saturnino et al. (2005), somente nos últimos 30 anos é que a espécie começou a ser mais pesquisada agronomicamente.

Um dos primeiros passos a serem delimitados diante a domesticação de uma espécie, é a determinação do nível de aptidão agrícola para a região específica, o que exige um levantamento, organização e análise de dados climáticos peculiares às suas necessidades (Sediyama et al., 2001).

A realização do zoneamento agrícola de uma região visa obter maiores informações sobre a adaptabilidade das culturas selecionadas e, sobretudo, proporcionar maior retorno dos investimentos a médio e longo prazos para os produtores contribuindo, desta forma, para uma agricultura racional e sustentável (Nunes et al., 2007).

Os elementos climáticos, em especial a temperatura e a pluviosidade, podem restringir o plantio e a condução das culturas em algumas regiões, contexto no qual o zoneamento agroclimático se constitui numa boa ferramenta para escolha de áreas de cultivo; Ressalta-se que, em Pernambuco, ainda não existem pesquisas que tratem do zoneamento do pinhãomanso no estado.

Com base no exposto, o presente trabalho teve como objetivo mapear áreas com aptidões agroclimáticas para o cultivo do pinhão-manso no estado de Pernambuco, baseandose nas exigências térmicas e hídricas da cultura.

\section{Material e MÉTOdos}

O estado de Pernambuco está localizado entre as Latitudes de $7^{\circ} 20^{\prime}$ a $9^{\circ} 29^{\prime} \mathrm{S}$ e Longitudes de $34^{\circ} 30^{\prime}$ a $41^{\circ} 21^{\prime} \mathrm{W}$, limitado pelos estados de Alagoas (ao sul), Paraíba (ao norte), Ceará (ao oeste) e o Oceano Atlântico (ao leste). O estado pernambucano é subdividido em cinco mesorregiões: Metropolitana do Recife (1), Zona da Mata (2), Agreste (3), Sertão (4) e São Francisco (5) como podem ser observadas na Figura 1.

Visando à realização do zoneamento agroclimático do pinhão-manso, utilizaram-se dados de precipitação pluviométrica e temperatura do ar (médias anuais) de 146 postos

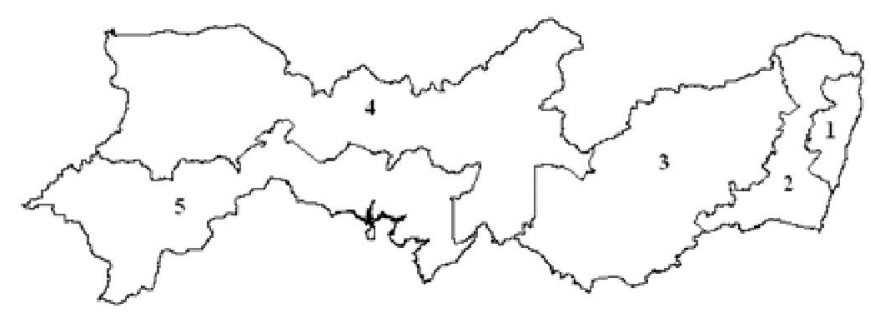

Figura 1. Mapa do estado de Pernambuco com as cinco mesorregiões: Região Metropolitana (1), Zona da Mata (2), Agreste (3), Sertão (4) e Sertão do São Francisco (5) 
meteorológicos de Pernambuco (Figura 2), dentre eles 73 postos com uma série acima de 30 anos de observações e outros 73 com uma série abaixo de 30 anos (porém maior que 20 anos) de observações para uma representatividade de dados melhor para o estado. Os dados climáticos mencionados são as médias anuais de temperatura e precipitação (SUDENE, 1990), disponíveis na página virtual da Unidade Acadêmica de Ciências Atmosféricas (UACA) da Universidade Federal de Campina Grande (UFCG).

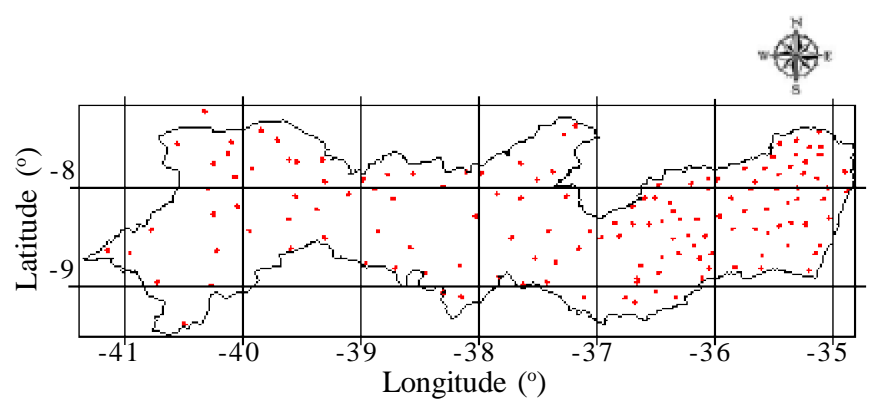

Figura 2. Distribuição dos postos meteorológicos no estado de Pernambuco

A estimativa das temperaturas médias do ar para os locais que dispunham apenas dos registros de chuva, foi realizada com o auxílio do Programa Estima_T (versão 2.0) desenvolvido por Cavalcanti et al. (2006) e que se encontra disponível gratuitamente na website da Unidade Acadêmica de Ciências Atmosféricas da Universidade Federal de Campina Grande. O programa utiliza um modelo de regressão múltipla quadrática para estimar a temperatura em localidades inseridas no Nordeste brasileiro em função da longitude, latitude e altitude. Tal modelo foi utilizado por Medeiros et al. (2009) e Silva et al. (2011) ao realizarem a estimativa da temperatura média do ar para diversas localidades em Pernambuco, objetivando a elaboração dos zoneamentos agroclimáticos para a Alpinia purpurata e pitangueira, no referido estado.

Caramori et al. (2001) e Wrege et al. (2005) esclarecem que é possível estimar a temperatura de um local com base em equações de regressão modeladas na altitude e latitude.

Alicerçados nas exigências hídricas e de temperatura estabelecidas por Henning (1996) e Saturnino et al. (2005), respectivamente, Gonçalves \& Sentelhas (2008) propuseram as seguintes classes de aptidão climática para a cultura do pinhão-manso (Tabela 1):

Tabela 1. Classes de aptidão climática para a cultura do pinhão-manso

\begin{tabular}{lcc}
\hline \multicolumn{1}{c}{ Regióes } & $\begin{array}{c}\text { Temperatura } \\
\left({ }^{\circ} \mathbf{C}\right)\end{array}$ & $\begin{array}{c}\text { Pluviosidade } \\
(\mathbf{m m})\end{array}$ \\
$\begin{array}{l}\text { Apta } \\
\text { Restrita por deficiência } \\
\text { hídrica }\end{array}$ & $18,0 \leq \mathrm{a} \leq 28,5$ & $>1000$ \\
$\begin{array}{l}\text { Restrita por deficiência } \\
\text { térmica (risco de geadas) }\end{array}$ & $<18,0 \leq \mathrm{a} \leq 28,5$ & $600 \leq \mathrm{a} \leq 1000$ \\
$\begin{array}{l}\text { Restrita por excesso de } \\
\text { temperatura }\end{array}$ & $>28,5$ & - \\
Inapta & - & - \\
\hline
\end{tabular}

Dallacort et al. (2010) também adotaram os mesmos limites realizando estudos de aptidão climática para o pinhão-manso na Região de Tangará da Serra, em Mato Grosso, e obtiveram resultados satisfatórios; em seguida, os limites térmicos e hídricos extrapolaram para todo o estado de Pernambuco encontrando, então, regiões semelhantes para as devidas classes de aptidão da cultura.

Os mapas de precipitação pluviométrica média anual, temperatura média anual e do zoneamento agroclimático para o pinhão-manso no estado de Pernambuco, foram elaborados pelo método de interpolação por krigagem; trata-se de um método de interpolação não linear que consiste na aplicação da krigagem ordinária sobre uma variável transformada, istoé, a variável resultante da aplicação de uma função indicadora dicotômica como utilizada por Castro et al. (2010) e geradas no programa computacional Surfer versão 7.0 (Golden Software).

Medeiros et al. (2009) e Toledo et al. (2009) também utilizaram o método da krigagem para confeccionarem, respectivamente, mapas de aptidão climática para a Alpinia purpurata em Pernambuco e pinhão-manso, no Espírito Santo.

\section{RESULTADOS E DISCUSSÃO}

De posse dos dados de precipitação pluviométrica e de temperatura do ar, foram gerados os mapas dos referidos elementos climáticos para o estado de Pernambuco (Figuras 3A e B).

Pode-se observar, na Figura (3A), que o Sertão Pernambucano apresenta, em sua maior área, faixas de precipitações entre

A.

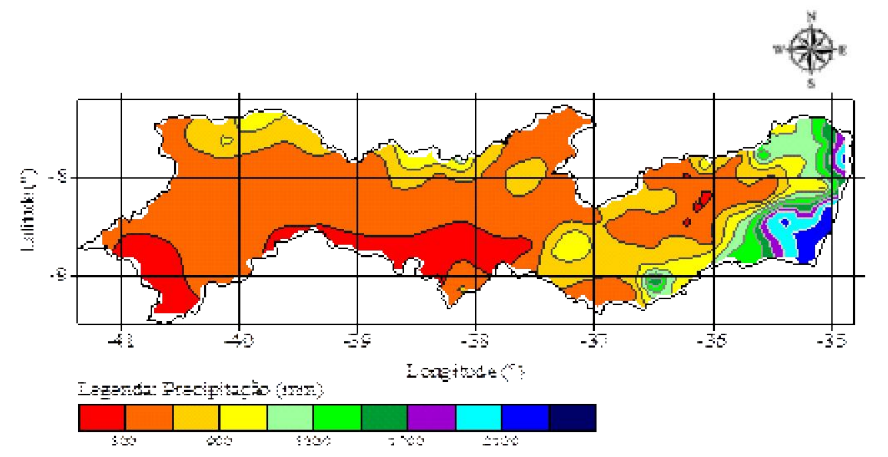

B.

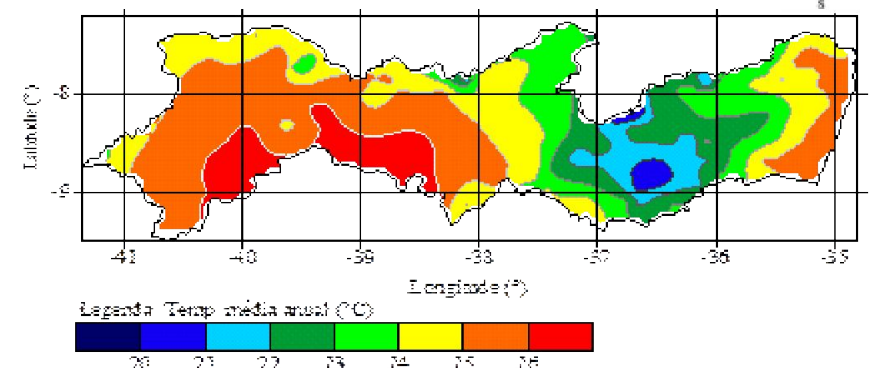

Figura 3. Variabilidade espacial da precipitação pluviométrica anual média $(A)$ e da temperatura anual média do ar (B) no estado de Pernambuco 
400 e $800 \mathrm{~mm}$ anuais, com os menores valores situados na mesorregião do São Francisco. Por sua vez, a mesorregião do Agreste apresenta precipitações da ordem de 600 a $1000 \mathrm{~mm}$ anuais em sua maior extensão porém é na Zona da Mata e no Litoral que se observam as maiores alturas pluviométricas anuais, com valores que vão de 1000 a 2265 mm, com destaque para o Litoral Sul. Excetuam-se, deste contexto, alguns Brejos de Altitude, como Bom Jardim $\left(\mathrm{LAT}=-35,58^{\circ}, \mathrm{LON}=-7,80^{\circ} \mathrm{e}\right.$ $\mathrm{ALT}=325 \mathrm{~m})$ no Agreste, com precipitação anual de $1485 \mathrm{~mm}$ e Triunfo $\left(\mathrm{LAT}=-38,11^{\circ}, \mathrm{LON}=-7,83^{\circ}\right.$ e ALT $\left.=1010 \mathrm{~m}\right)$ no Sertão, precipitação anual de $1231 \mathrm{~mm}$.

Para Cabral et al. (2004) as chuvas nas regiões de alguns brejos pernambucanos apresentam características orográficas, sofrendo influência do relevo e das massas de ar úmido, que se deslocam pelos vales dos rios e encontram, nos brejos, condições propícias para condensação.

Apesar da grande variabilidade de chuvas em Pernambuco, as atividades produtivas no estado não sofrem impactos adversos das condições médias da meteorologia, quer de baixa, média ou alta pluviosidade, mas sim da sua variabilidade temporal e espacial, ocasionando enchentes em áreas da Zona da Mata e Região Metropolitana do Recife, além de longos períodos de estiagem no Sertão.

Observa-se, na Figura 3B, que as maiores faixas de temperatura média do ar se situam ao redor da mesorregião do Sertão do São Francisco. Por outro lado, as isolinhas com os menores valores de temperatura média giram dentro da mesorregião do Agreste pernambucano; o principal motivo para menores faixas de temperatura nesta região está atribuído, provavelmente, ao fator climático "relevo", uma vez que é na região do Agreste que se encontra boa parte dos "Brejos de Altitudes" de Pernambuco, como Porção (LAT = -36,70, LON $=-8,18^{\circ}$ e $\left.\mathrm{ALT}=1035 \mathrm{~m}\right)$, Buíque $\left(\mathrm{LAT}=-37,16^{\circ}, \mathrm{LON}=-8,61^{\circ}\right.$ e ALT $=798 \mathrm{~m})$ e Brejão $\left(\mathrm{LAT}=-36,50^{\circ}, \mathrm{LON}=-9,05^{\circ}\right.$ e $\mathrm{ALT}=$ $620 \mathrm{~m}$ ) dentre outros.

Segundo Cabral et al. (2004), nos Brejos de Altitude localizados na região Agreste de Pernambuco, o microclima é diferenciado do entorno; as temperaturas são mais amenas e, por efeito orográfico, a pluviosidade é bastante superior à das áreas circunvizinhas.

Andrade et al. (2007) salientam que a temperatura tem estreita relação com a altitude e a latitude. De maneira geral, a cada 100 $\mathrm{m}$ de elevação a temperatura decresce, em média, $0,65^{\circ} \mathrm{C}$. Esta relação de decréscimo é denominada gradiente adiabático úmido e é frequentemente utilizada como critério para estimar temperaturas médias para locais em que não se dispõe de estações meteorológicas.

A Figura 4 apresenta as médias anuais de temperatura (A) e precipitação pluviométrica (B) para as 146 localidades analisadas em Pernambuco.

Observado, na Figura 4A, que a temperatura média do ar variou de $19,5^{\circ} \mathrm{C}$ (na cidade de Porção, $\mathrm{LAT}=-36,70^{\circ}, \mathrm{LON}=$ $-8,18^{\circ}$ e $\mathrm{ALT}=1035 \mathrm{~m}$ ) a $26,4^{\circ} \mathrm{C}$ (no Município de Floresta, $\mathrm{LAT}=-8,60^{\circ}, \mathrm{LON}=-38,58^{\circ}$ eALT $=317 \mathrm{~m}$ ). De acordo com a proposição de Saturnino et al. (2005), a faixa ótima para o cultivo do pinhão-manso se situa entre 18 e $28,5{ }^{\circ} \mathrm{C}$ não havendo, assim, no estado de Pernambuco, restrições de ordem térmica para o desenvolvimento da referida cultura, mesmo nas regiões

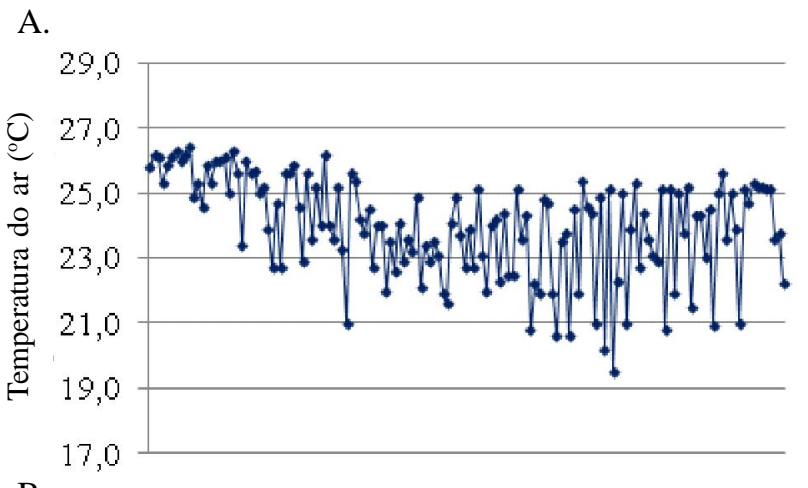

B.

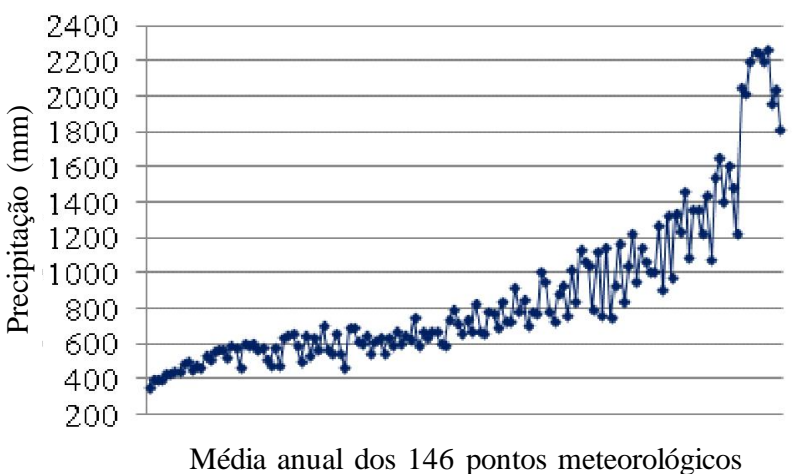

Figura 4. Temperaturas (A) e precipitações (B) (médias anuais) relativas aos 146 postos analisados em Pernambuco

de maiores altitudes. Gonçalves \& Sentelhas (2008) constataram, ao analisar o regime térmico da Bahia, que naquele estado também não houve restrição para o cultivo do pinhão-manso, no que se refere à temperatura do ar.

Constata-se, na Figura 4B, que a precipitação pluviométrica em Pernambuco variou entre 350 (na localidade de Pau Ferro em Petrolina, $\mathrm{LAT}=-40,73^{\circ}, \mathrm{LON}=-8,95^{\circ}$ e $\left.\mathrm{ALT}=385 \mathrm{~m}\right)$ e 2265 $\mathrm{mm}$ (em Rio Formoso, $\mathrm{LAT}=-35,15^{\circ}, \mathrm{LON}=-8,66^{\circ}$ e ALT $=39$ $\mathrm{m})$. A grande variação no total de chuvas anual no estado faz com que existam três zonas distintas de favorabilidade ao cultivo do pinhão-manso.

Conforme a exigência hídrica, em regiões tropicais esta oleaginosa se desenvolve bem onde a precipitação anual esteja acima de $600 \mathrm{~mm}$, sendo que o nível ideal de chuva para atingir altas produtividades estaria acima dos $1000 \mathrm{~mm}$ anuais; com uma precipitação anual menor que $600 \mathrm{~mm}$, a produção é drasticamente afetada (Henning, 1996; Gonçalves \& Sentelhas, 2008).

De acordo com as exigências hídricas da cultura do pinhãomanso, das 146 localidades analisadas em Pernambuco, 42 se apresentaram inaptas, 63 em condições restritas (por deficiência hídrica) e 41 em condições de aptidão.

A ampla favorabilidade térmica para o cultivo do pinhãomanso, integrada às condições hídricas, resultou no zoneamento agroclimático do pinhão-manso para o estado de Pernambuco (Figura 5).

Observando a Figura 5, nota-se que o mapa do zoneamento agroclimático do pinhão-manso em Pernambuco, possui certa semelhança com o mapa das Mesorregiões do estado, fato que está provavelmente relacionado com a distribuição das chuvas dentro do estado. 


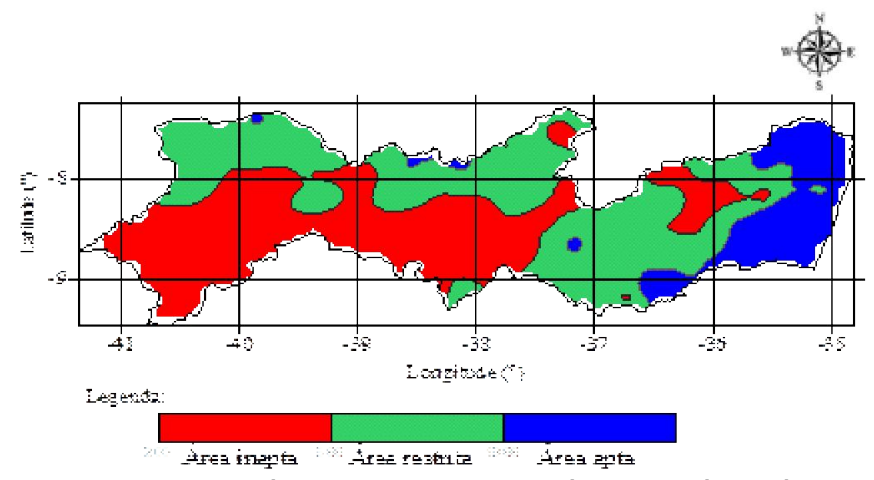

Figura 5. Mapa do zoneamento agroclimático do pinhãomanso em Pernambuco

Segundo Varejão-Silva \& Cezar-Barros (2001) há certo grau de incerteza na posição geográfica do traçado das linhas que separam as diferentes classes de aptidão climática, em razão da distribuição espacial dos postos pluviométricos no estado de Pernambuco, a qual ainda está bem aquém do desejável, deixando algumas áreas sem qualquer informação, dificultando o traçado das linhas, ou seja, do limite das faixas de aptidão estimadas.

Ainda analisando a Figura 5, pode-se observar que as áreas aptas (que correspondem aproximadamente a 14,92\% da área total do estado) ao cultivo do pinhão-manso, estão presentes, em maior extensão, na Zona da Mata e litoral Pernambucano, apesar de apresentarem pequenas exceções, como é o caso de algumas áreas situadas em alguns Brejos de Altitude localizados no agreste e no sertão do estado. Referidas regiões apresentam regime pluviométrico satisfatório (acima de $1000 \mathrm{~mm}$ anuais) para que o pinhão-manso consiga produzir como cultura de sequeiro. Essas áreas são consideradas aptas, por apresentar temperatura e chuva necessárias para que a cultura possa produzir satisfatoriamente.

As Mesorregiões do Agreste e Sertão apresentam vasta extensão territorial, como regiões de aptidão restrita para o cultivo desta oleaginosa visto que não satisfazem todas as condições climaticamente necessárias para uma produção satisfatória do pinhão-manso. Nessas regiões as chuvas anuais se situam entre 600 e $1000 \mathrm{~mm}$ permitindo o cultivo da cultura porém com restrições à produtividade como cultura de sequeiro. Toda essa região foi considerada marginal por deficiência hídrica. Gonçalves \& Sentelhas (2008) recomendaram, para regiões com essa faixa de pluviosidade, a utilização de irrigação suplementar, desde que seja economicamente viável. As áreas classificadas como restrita abrangem cerca de $44,26 \%$ da área total do estado.

Foi classificado, como áreas inaptas ao cultivo do pinhãomanso como cultura de sequeiro, o total de 40,82\% distribuídos principalmente na Mesorregião do Sertão do São Francisco. Apresentam-se ainda, como áreas inaptas, uma parte da Microrregião do Vale do Ipojuca, no Agreste, certa extensão das Microrregiões do Moxotó e Salgueiro e, ainda, uma pequena área ao Norte da Microrregião do Pajeú, essas três pertencentes ao Sertão Pernambucano; tais regiões apresentam deficiência hídrica bastante acentuada e precipitações abaixo de $600 \mathrm{~mm}$ anuais. A baixa pluviosidade se apresenta como fator limitante para a produção do pinhão-manso.
Não foram diagnosticadas áreas restritas por deficiência térmica nem por deficiência térmica e hídrica.

\section{ConClusÕes}

1. Em se tratando do zoneamento agroclimático para a cultura do pinhão-manso em Pernambuco, das 146 localidades analisadas, foi possível diagnosticar, no estado, áreas com as seguintes características climáticas:

- Áreas Aptas: 41 localidades no estado se apresentaram aptas à produção do pinhão-manso como cultura de sequeiro totalizando uma área de aproximadamente $14,92 \%$;

- Áreas restritas: 63 localidades foram classificadas como restritas, perfazendo uma área em torno de 44,26\%;

- Áreas inaptas: 42 localidades foram diagnosticadas como inaptas, abrangendo uma área de 40,82\%.

2. Em se considerando os limites empregados no Brasil para definição da condição de aptidão hídrica para a cultura do pinhão-manso, qual seja, precipitação anual média de $1000 \mathrm{~mm}$, ficou evidenciado que as áreas aptas compreendem áreas que, tradicionalmente, são ocupadas com a cana-de-açúcar, em Pernambuco.

\section{LITERATURA CITADA}

Ambrozim, A. R. P.; Kuri, S. E.; Monteiro, M. R.Corrosão metálica associada ao uso de combustíveis minerais e biocombustíveis. Química Nova, v.32, p.1910-1916, 2009.

Andrade, G. A.; Caramori, P. H.; Caviglione, J. H.; Dalziza de Oliveira, D. de; Ribeiro, A. M. de A. Zoneamento agroclimático para a cultura do pinhão-manso (Jatropha curcas) no estado do Paraná. Revista Brasileira de Agrometeorologia, v.15, p.178-183, 2007.

ANP - Agência Nacional do Petróleo, Gás Natural e Biocombustíveis. Superintendência de Planejamento e Pesquisa. ANP, 2012. 4p. Boletim Eletrônico, 53.

Arruda. F. P.; Beltrão. N. E. M.; Andrade. A. P.; Pereira. W. E.; Severino. L. S. Cultivo de Pinhão Manso (Jatropha curcas L.) como alternativa para o semi-árido nordestino. Revista Brasileira de Oleaginosas e Fibrosas, v.8, p.789-799, 2004.

Cabral, J. J. P.; Braga, R. A. P.; Montenegro, S. M. G. L.; Campello, M. S. C.; Lopes Filho, S. Recursos hídricos e os brejos de altitude. In: Porto, K. C.; Cabral, J. J. P., Tabarelli, M. Brejos de altitude em Pernambuco e Paraíba: História natural, ecologia e conservação. Brasília: Ministério do Meio Ambiente, 2004. cap. 4, p.31-48. Série Biodiversidade, 9.

Caramori, P. H; Caviglione, J. H.; Wrege, M. S.; Gonçalves, S. L.; Faria, R. T. de; Androcioli Filho, A.; Sera, T.; Chaves, J. C. D.; Koguishi, M. S. Zoneamento de riscos climáticos para a cultura de café (Coffea arabica L.) no estado do Paraná. Revista Brasileira de Agrometeorologia, v.9, p.486-494, 2001.

Castro, F. da S.; Pezzopane, J. E. M.; Cecílio, R. A.; Pezzopane, J. R. M.; Xavier, A. C. Avaliação de desempenho dos diferentes métodos de interpoladores para parâmetros do balanço hídrico climatológico. Revista Brasileira de Engenharia Agrícola e Ambiental, v.14, p.871-880, 2010. 
Cavalcanti, E. P.; Silva, V. de P. R. da; Sousa, F. A. S. Programa computacional para estimativa da temperatura do ar para a Região Nordeste do Brasil. Revista Brasileira de Engenharia Agrícola e Ambiental, v.10, p.140-147, 2006.

Dallacort, R.; Martins, J. A.; Inoue, M. H.; Freitas, P. S. L.; Krause, W. Aptidão agroclimática do pinhão-manso na região de Tangará da Serra, MT. Revista Ciência Agronômica, v.41, p.333-379, 2010.

Drumond, M. A.; Santos, C. A. F.; Oliveira, V. R. de; Martins, J. C.; Anjos, J. B. dos; Evangelista, M. R. V. Desempenho agronômico de genótipos de pinhão manso no Semiárido pernambucano. Ciência Rural, v.40, p.44-47, 2010.

Gonçalves, M. B.; Sentelhas, P. C. Zoneamento agroclimático para a cultura do pinhão-manso no estado da Bahia, objetivando a produção de biocombustível. In: UNESCO, MBC, RECyT/Mercosul, CNPq, Petrobras. (Org.) Biocombustível para o Mercosul: ed.2008. Brasília: Ministério da Ciência e Tecnologia, 2008. p.121-140.

Henning, R. Combating desertification: The jatropha project of Mali, West África. Aridlands Newsletter: The CCD, Part I: Africa and the Mediterranean, n.40, fall/winter. Tucson: University of Arizona, 1996. s.p. http://ag.arizona.edu/oals/ ALN/aln40/jatropha.html

Lima Filho, D. de O.; Sogabe, V. P.; Calarge, T. C. C. Mercado do biodiesel: Um panorama Mundial. Espacios, v.29, p.5-27, 2008.

Medeiros, S. R. R.; Moura, G. B. A.; Giongo, P. R.; Silva, A. P. N. Potencial agroclimático para a Alpinia purpurata, no estado de Pernambuco. Revista Brasileira de Engenharia Agrícola eAmbiental, v.13, p.165-169, 2009.

Nunes, E. L.; Amorim, R. C F.; Sousa, W. G.; Ribeiro, A.; Senna, M. C. A.; Leal, B. G. Zoneamento agroclimático da cultura do café para a bacia do Rio Doce. Revista Brasileira de Meteorologia, v.22, p.297-302, 2007.

Roscoe, R.; Richetti, A.; Maranho, E. Análise de viabilidade técnica de oleaginosas para produção de biodiesel em Mato Grosso do Sul. Revista de Política Agrícola, ano XVI, n.01, p.48-59. 2007.
Sato, M.; Bueno, O. de C.; Esperancini, M. S. T.; Frigo, E. P. A cultura do pinhão-manso (Jatropha curcas L.): Uso para fins combustíveis e descrição agronômica. Revista Varia Scientia, v.07, p.47-62, 2007.

Saturnino, H. M.; Pacheco, D. D; Kakida, J.; Tominaga, N.; Gonçalves, N. P. Cultura do pinhão-manso (Jatropha curcas L.). Belo Horizonte: EPAMIG, 2005. v.26, p.44-78. Informe Agropecuário, 229.

Sediyama, G. C. Melo Júnior, J. C. F.; Santos, A. R.; Ribeiro, A. Costa, M. H.; Hamakawa, P. J.; Costa, J. M. N.; Costa, L. C. Zoneamento agroclimático do cafeeiro (Coffea arabica L.) para o estado de Minas Gerais. Revista Brasileira de Agrometeorologia, v.09, p.501-509, 2001.

Silva, A. O. da; Silva, A. P. N da; Moura, G. B. de A.; Lopes, P. M. O.; Medeiros, S. R. R de. Zoneamento agroclimático da pitangueira para três cenários distintos de precipitação pluvial em Pernambuco. Revista Caatinga, v.24, p.104-115, 2011.

SUDENE - Superintendência do Desenvolvimento do Nordeste. Dados pluviométricos mensais do Nordeste. Recife: SUDENE, 1990 Série Pluviometria 1 a 10.

Toledo, J. V.; Martins, L. D.; Klippel, V. H.; Pezzopane, J. E. M.; Tomaz, M. A.; Amaral, J. F. T dos. Zoneamento agroclimático para a cultura do pinhão-manso (Jatropha curcas L.) e da mamona (Ricinus communis L.) no estado do Espírito Santo. Agropecuária Científica no Semi-Árido, v.5, p.41-51, 2009.

Varejão-Silva, M. A.; Cezar-Barros, A. H. Zoneamento de aptidão climática do estado de Pernambuco para três distintos cenários pluviométricos. Recife: COTEC/Data Agros Informática \& Informações Agrícolas/Secretaria de Produção Rural e Reforma Agrária de Pernambuco. 2001. 38p. Relatório Técnico

Wrege, M. S.; Caramori, P. H.; Gonçalves, A. C. A.; Bertonha, A.; Ferreira, R. C.; Caviglione, J. H.; Faria, R. T. de; Freitas, P. S. L. de; Gonçalves, S. L. Regiões potenciais para cultivo da cana-de-açúcar no Paraná, com base na análise do risco de geadas. Revista Brasileira de Agrometeorologia, v.13, p.113-122, 2005. 